Central Washington University

ScholarWorks@CWU

All Faculty Scholarship for the College of the Sciences

8-7-1995

\title{
Phononic band structure in a mass chain
}

Samantha Parmley

Tom Zobrist

Terry Clough

Anthony Perez-Miller

Mark Makela

See next page for additional authors

Follow this and additional works at: https://digitalcommons.cwu.edu/cotsfac

Part of the Elementary Particles and Fields and String Theory Commons 
Authors

Samantha Parmley, Tom Zobrist, Terry Clough, Anthony Perez-Miller, Mark Makela, and Roger Yu 


\title{
Phononic band structure in a mass chain
}

\author{
Samantha Parmley, Tom Zobrist, Terry Clough, Anthony Perez-Miller, Mark Makela, \\ and Roger Yu \\ Department of Physics, Central Washington University, Ellensburg, Virginia 98926
}

(Received 13 February 1995; accepted for publication 24 May 1995)

\begin{abstract}
The vibrational properties of a finite one-dimensional string-mass chain are studied experimentally and theoretically. In the experiment both normal mode analysis and pulse analysis are used to obtain the eigenfrequencies of the string-mass chain. The theoretical predictions are made based upon the numerical solution to the wave equation. The phononic band structure for a periodically massed string as well as Anderson localized gap modes for a disordered system are found. The theoretical and experimental results match satisfactorily well. (C) 1995 American Institute of Physics.
\end{abstract}

As a result of the Bloch theory, the eigenstates of an electron in a strictly periodic potential are extended and the electron energy levels form allowed energy bands separated by forbidden energy gaps. The analogy between the electronic band structure and the optical band structure has been explored recently. A three-dimensional face-centered-cubic dielectric structure ("photonic crystal") was introduced by Yablonovitch et al. ${ }^{1}$ In the photonic crystal, transmission bands and gaps were conclusively identified. A mechanical wave propagating in a periodic medium also resembles the dynamics of an electron in a crystal. More recently, the calculation of an "acoustic" or "phononic" band structure in a three-dimensional periodic composite of two materials with different elastic properties was carried out by Kushwaha et al. $^{2}$

In this letter we report the results of detailed theoretical predictions and experimental measurements on the acoustic properties of a finite regular and irregular one-dimensional string-mass chain. The theoretical predictions are made based upon the numerical solution of the wave equation. In the numerical simulation the Fourier expansion was used to transform the differential wave equation into a matrix equation. With the formalism developed in this research it is possible to determine the eigenvalues and eigenfunctions to any degree of accuracy. There is no restriction on the distribution and the magnitude of the masses; they can be completely random. Therefore, the approach may be employed to simulate not only perfect one-dimensional crystals, but also disordered crystals, quasicrystal, and amorphous structures.

On the experimental side, techniques of studying the dynamical property of a microscopic electronic system or a macroscopic mechanical system can be characterized into two categories: normal mode analysis (NMA) and pulse analysis (PA). In the NMA, the transmitted amplitude through the system is recorded while the exciting (driving) frequency is being swept. The response of the system is expected to be enhanced when the exciting frequency is near a resonant (eigen) frequency. In the PA, a pulse which is rich in frequency, is received by the string system. The subsequent vibration of the system is recorded. Then the spectrum is obtained by carrying out a Fourier transform of the time series. The spectrum reveals all the important information about the eigenproperties of the system by peaking its value at eigenfrequencies. In the experiments reported here, both methods were used and the results are compared.

We consider the standing (eigen) wave equation of a stretched and clamped string with loaded masses

$$
\left(\omega_{n}\right)^{2} \sigma(x) u_{n}(x)+T_{0} u_{n}(x)=0,
$$

here, $T_{0}$ is the tension in the string, $\sigma(x)$ is the mass density which takes into account the sizes and positions of loaded masses as well as the string, and $\omega_{n}$ and $u_{n}(x)$ are the eigenfrequencies and eigenwave functions, respectively. The above equation was solved numerically using a software package in the IMSL Math/Library. ${ }^{4}$ The transient vibrational property of the loaded string can be described by the eigenwave functions found above. The time evolution of plucked string $\Gamma(x, t)$ is expressed by the superposition of the eigenstates, $u_{n}(x) e^{i \omega_{n} t}$

$$
\Gamma(x, t)=\sum_{n} d_{n} u_{n}(x) e^{i \omega_{n} t},
$$

where, the expansion coefficients $d_{n}$ are determined by the initial displacement (plucked form) of the string $\Gamma(x, 0)$,

$$
d_{n}=\int_{0}^{L} \Gamma(x, 0) u_{n}(x) d x .
$$

The time series $\Gamma(x, t)$ for a fixed point $x=a$, is in general quite noisy except when the string is vibrating at a resonant frequency. However, the Fourier transform $S(\omega)$, of $\Gamma(a, t)$ is rich in physical properties and less difficult to interpret. The spectrum $S(\omega)$ is defined as

$$
S(\omega)=\frac{1}{2 \pi} \int_{-\infty}^{+\infty} \Gamma(a, t) e^{-i \omega t} d t .
$$

By substituting Eq. (2) into Eq. (4), we have

$$
\begin{aligned}
S(\omega) & =\sum_{n} d_{n} u_{n}(a) \frac{1}{2 \pi} \int_{-\infty}^{+\infty} e^{-i\left(\omega-\omega_{n}\right) t} d t \\
& =\sum_{n} d_{n} u_{n}(a) \delta\left(\omega-\omega_{n}\right) .
\end{aligned}
$$

It is obvious that spectrum $S(\omega)$ peaks at resonant frequencies $\omega_{n}$. In practice, the damping in the string is not negligible, then the delta function $\delta\left(\omega-\omega_{n}\right)$ is broadened by damping. The oscillator strength for each eigenstate depends on the initial expansion coefficient $d_{n}$. 

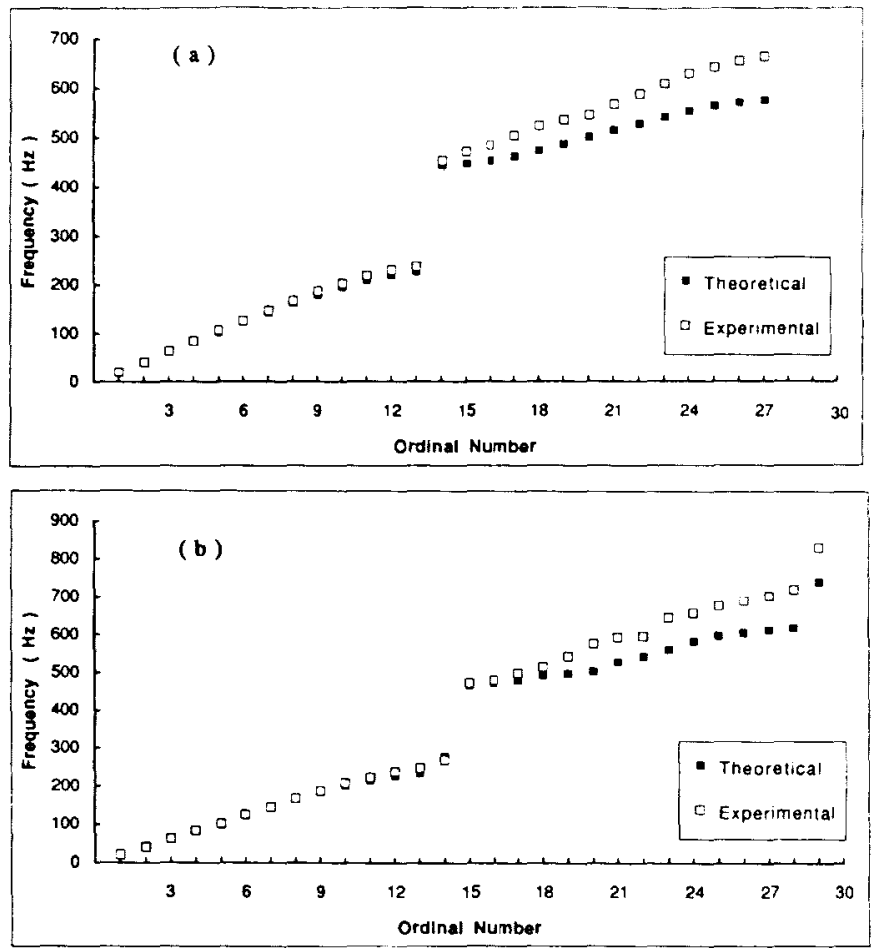

FIG. 1. (a) and (b) show the theoretical and experimental resonant frequencies of the periodic (random) 13-mass string as a function of the ordinal number $n$.

In the experiment, we use a TekTronix FG 504 Sweeping Function Generator to produce a sinusoidal wave, which we can either set to specific frequencies, or sweep through a variable range of frequencies. The signal is then run through an amplifier to an ordinary audio speaker. The string is physically attached to the core of the speaker, so that the string and the speaker vibrate at the same frequency. We have devised two methods, corresponding to the two different analyses, for recording the data from the pickups. One is to run the ac signal from the pickup through a rectifier to produce a de signal. This signal is then run into a SargentWelch $x-y$ recorded which will produce a hard copy graph of amplitude versus frequency for a range of frequencies swept through by the function generator. This method allows us to perform eigenmode analysis. The second method of recording the data is based on the use of a computer driven digitizer. The ac signal from the pickup is run through the linear amplifier, then through a low-pass filter and into the digitizer. The digitized data enable us to analyze the real time series in the frequency domain by fast Fourier transformation (FFT). The power spectrum will exhibit peaks which can be used to identify the eigenfrequencies of the system. This method works the best for the pulse analysis, and for simply plucking the string.

We now present the results of ordered and disordered systems in a parallel fashion; figures for different systems are shown together for comparison. The NMA resonant frequencies, wave functions, and spectrum for a periodic system are displayed in Figs. 1, 2, and 3, respectively, by parts (a), whereas their counterparts for the disordered system are shown by parts (b). Thirteen masses are evenly spaced on a

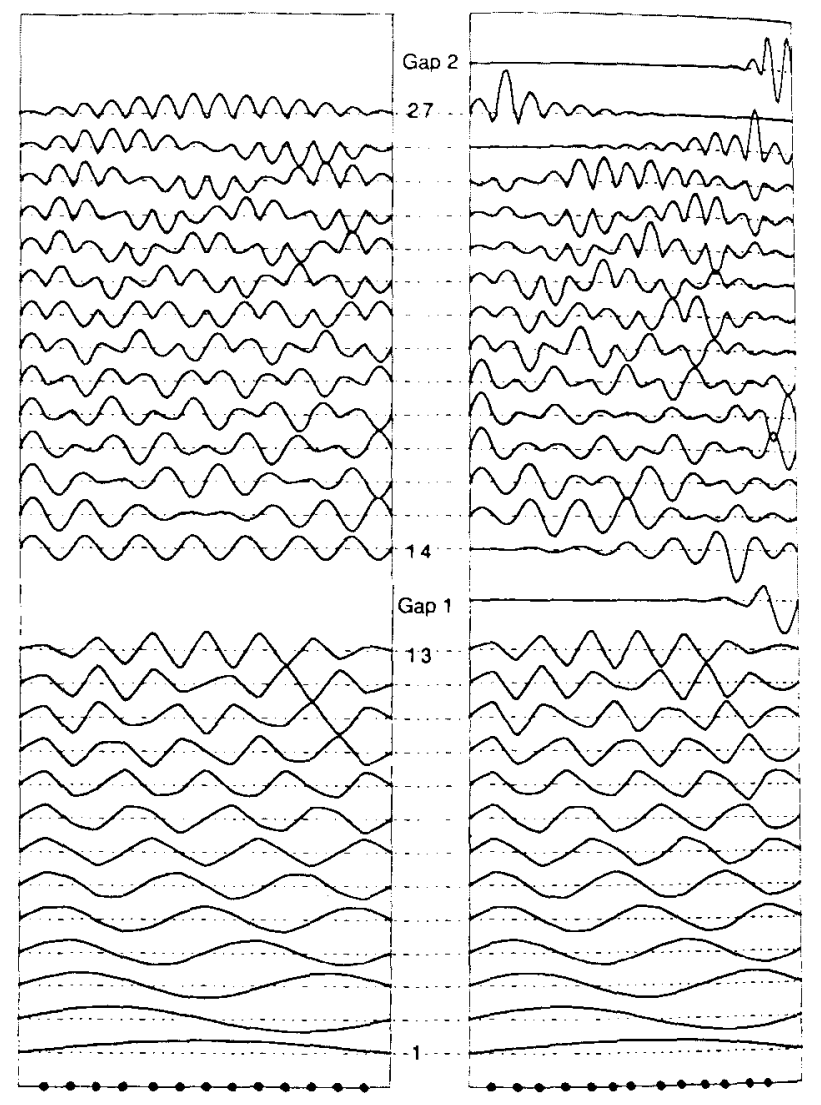

FIG. 2. The eigenfunctions of the lowest two bands of the periodic lleft hand-side column) and the random (right-hand side column) 13-mass string. The positions of masses are marked.

string for the periodic system. About $10 \%$ random variation in mass separation is incorporated in the disordered string. It can be seen in Fig. 1 that the NMA data and the theoretical prediction match quite well for both systems, especially in the lowest bands. The dispersion is especially interesting in two limited; i.e., long wave length (small $n$ ) and short wave length (large $n$ ). At the Brillouin zone boundary, $n=13$, the group velocity of the system, which equals the derivative of frequency with respect to the wave vector $\mathbf{k}$ (or $\mathbf{n}$ ), is almost zero. It is clearly seen in the eigenfunctions shown in Fig. 2(a) that when $n=13$, all the masses are vibrating out of phase and the wavelength $\lambda=2 a$, so that the wave of the system looks more like a standing wave with zero group velocity. At the other end of the Brillouin zone boundary $n=1$, the wavelength $\lambda$ is large. One can view the system as a continuum and the string wave becomes an acoustic wave. ${ }^{5}$ In the second band, significant discrepancy between the theoretical and experimental data is observed. We attribute the upshift in experimental resonant frequencies to the stiffness of the wire. The change in the tension due to the stiffness can be approximated by a simple equation, $T(x)$ $\approx T_{0}+(Y A / 2)(\partial u / \partial x)^{2}$, here $Y$ and $A$ are the Young's modulus and the cross-section area of the wire, respectively. The second (nonlinear) term of the above equation increases the tension, and so shifts the frequency upward. In our theoretical calculations, the nonlinear effect was neglected. Further study of our system using a wire with lower stiffness has yielded experimental data much closer to the theoretical 

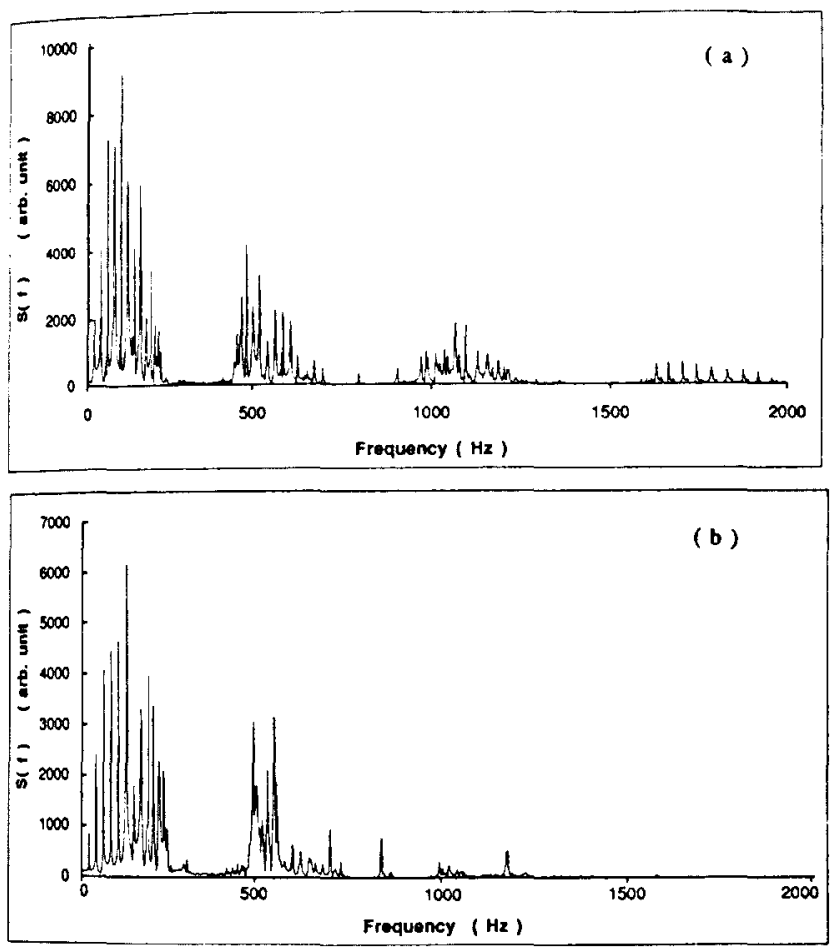

FlG. 3. Spectra for the (a) periodic and (b) random 13-mass string. The spectra are obtained by Fourier transforming the time series of vibration at a fixed point on the string.

work. The experimental data in Fig. 1 show a nonzero group velocity (finite slope) near $n=14$. The structure in the middle of the band $n=20$, displays a smaller group velocity. Maynard and his co-workers ${ }^{6}$ have observed regions of low response in the second band in a similar measurement. They attributed the effect to the $\sim 13 \%$ variation in the size of the commercial lead masses. We believe that the same argument may be applied to our data shown in Fig. 1.

The dispersion relation for the random system, shown in Fig. 1(b) resembles that of the periodic system in the lowest band. The wave functions of the lowest band for both systems are all extended [see Figs. 2(a) and 2(b)]. In a disordered system, the wave functions generally have three types of characteristics: (i) extended, (ii) localized, and (iii) critical which is the combination of (i) and (ii) ${ }^{7}$ In Fig. 2(b), the gap mode (GAP 1) is strongly localized ${ }^{8}$ whereas the lowest and the highest states in the second passing band $(n=14,27)$ are critical. The gap mode existing in between the second and third bands (GAP 2) is also highly localized.

In the spectrum $S(\omega)$ [Fig. 3(a)], four frequency bands are distinguishable for the periodic system. In the first and second band, one can actually see 13 and 14 peaks, respectively, corresponding to 13 and 14 eigenfrequencies in each band. The first passing band weighs more heavily in oscilla- tor strength than the higher bands, it is because the size of the plucked initial wave form is in the same order of magnitude as wavelengths of the lowest band. One of the theoretical (linear theory) predictions for the mechanical system considered here is that the band width of higher bands become narrower, which is just the opposite of an electronic band structure. By studying the theoretical wave forms in Fig. 2(a), we have found that the amplitude of vibration of the masses becomes smaller in the higher band. It is clear that the less the motion of a point mass, the weaker the coupling between the string segments on both sides of the mass; if the mass does not move the coupling is zero. As a result of weaker coupling, the bandwidth decreases. In contrast, the measured frequency bandwidth does not decrease significantly because of the upshift in frequencies due to the nonlinearity of the system.

For the disordered system, three bands are visible in the spectrum shown in Fig. 3(b). The distinct feature in the spectrum is the active response in the gaps. Immediately above the lowest passing band and below the second band, weak peaks corresponding to the localized gap mode can be easily observed. The gap mode near $750 \mathrm{~Hz}$ in the second gap [ $n=28$ in Figs. 1(a) and 2(b)] has relatively strong oscillator strength because its wave function is localized near the drive situated at the left end of the string, therefore this mode is more easily excited.

In summary, we have numerically and experimentally studied the vibrational properties of a string loaded with masses. For a periodically loaded string we have found that the resonant frequencies form bands whose wave functions are extended. In contrast to the periodic system, strongly localized modes existed in the gaps or near the edges of the frequency bands are found in a randomly loaded string. The physical properties found in the classical system are reminiscent of many important quantum mechanical effects. The experiment presented here provides vivid visualization of those effects on a macroscopic scale.

One of the authors (S.P.) appreciates the support from the Council on Undergraduate Research Summer Opportunities for Research (CURSOR) Fellowship. R.Y. thanks the financial support from the College of Graduate Studies and Research at Central Washington University.

${ }^{1}$ E. Yablonovitch, J. J. Gmitter, and K. M. Leung, Phys. Rev. Lett. 67, 2295 (1991).

${ }^{2}$ M. Kushwaha, P. Haleve, L. Dobrzynski, and B. Diafari-Rouhani, Phys. Rev. Lett. 71, 2022 (1993).

${ }^{3}$ R. Yu, Phys. Rev. B 47, 15692 (1993); W. Glessner and R. Yu, J. Appl. Phys. 73, 7040 (1993).

${ }^{4}$ IMSL Math/Library (Visualnumerics, Houston, 1989).

${ }^{5}$ G. P. Srivastava, The Physics of Phonons (Adam Hilger, Bristol, Philadelphia and New York, 1990).

${ }^{6} \mathrm{~S}$. He and J. Maynard, Phys. Rev. Lett. 57, 3171 (1986).

${ }^{7}$ J. Lu, T. Odagaki, and J. Birman, Phys. Rev. B 33, 4809 (1986).

${ }^{8}$ P. Anderson, Phys. Rev. 109, 1492 (1958). 